\title{
Generation and Relaxation of Microstrains in GaN Nanocrystals under Extreme Pressures
}

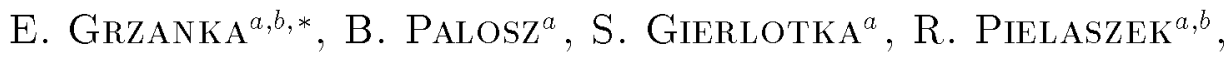

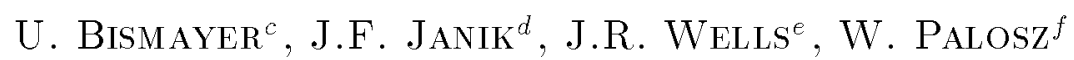 \\ AND F. PORSCH ${ }^{g}$ \\ ${ }^{a}$ High Pressure Research Center UNIPRESS \\ Sokołowska 29/37, 01-142 Warsaw, Poland \\ ${ }^{b}$ Institute of Experimental Physics, Warsaw University, Warsaw, Poland \\ ${ }^{c}$ Mineral-Petrographisches Institut, University of Hamburg, Hamburg, Germany \\ ${ }^{d}$ University of Mining and Metallurgy, Cracow, Poland \\ ${ }^{e}$ Department of Chemistry, Duke University, Durham, USA \\ ${ }^{f}$ USRA/NASA-Marshall Space Flight Center, Huntsville, Alabama, USA \\ ${ }^{g}$ Mineralogisch-Petrologisches Institut der Rheinischen \\ Friedrich-Wilhelms-Universität Bonn, Bonn, Germany
}

\begin{abstract}
Nanocrystalline powders of $\mathrm{GaN}$ with grain sizes ranging from 2 to $30 \mathrm{~nm}$ were examined under high external pressures by in situ diffraction techniques in a diamond anvil cell at DESY (HASYLAB, Station F3). The experiments on densification of pure powders under high pressure were performed without a pressure medium. The mechanism of generation and relaxation of internal strains and their distribution in nanoparticles was deduced from the Bragg reflections recorded in situ under high pressures at room temperature. The microstrain was calculated from the full-width at half-maximum (FWHM) values of the Bragg lines. It was found that microstrains in GaN crystallites are generated and subsequently relaxed by two mechanisms: generation of stacking faults and change of the size and shape of the grains occurring under external stress.
\end{abstract}

PACS numbers: $61.46 .+\mathrm{w}, 81.07 . \mathrm{Wx}_{\mathrm{x}}$

*corresponding author; e-mail: elesk@unipress.waw.pl 


\section{Introduction}

In a polycrystalline powder densified without a pressure medium (i.e. under so-called isostatic pressure conditions) strong microstrains may exist at the contacts between individual grains. In a porous material the applied external load is transferred only through relatively small contact areas between the adjacent grains. As a consequence high local stresses develop in the material. With an increase in the external pressure the increase in the local strains may be expected to diminish due to the increase in the total contact area (necking) between the particles. This may be accompanied by changes in the grain shapes and sizes leading to generation of dislocations where the local strains are distributed more evenly than those at the contact areas.

The generation and/or movement of dislocations may lead to local changes of relative positions of the atomic planes. In close packed structures this may lead to a creation of stacking faults. In the case of the wurtzite structure the formation of stacking faults is equivalent to the creation of a cubic-like stacking of layers in the parent sequence of hexagonal layers. Since the strains are relaxed through generation of stacking faults, the number of faults should be proportional to the applied external stress. However, due to its size, a single nanocrystallite can accommodate only a limited number of dislocations and, thus, only a limited number of stacking faults can form in the nanoparticle. With a further increase in the external stress the relaxation of the strains could occur through other mechanisms, e.g. by changing the shape or breaking the individual grains into smaller crystallites, which leads to a more compact packing of the particles.

This work is dedicated to the analysis of the mechanism of relaxation of strains in GaN nanocrystals subjected to high external stresses. Under normal conditions the stable crystallographic phase of bulk GaN is the hexagonal-wurtzite lattice (2H polytype) and micrometer size grains of GaN always show a perfect wurtzite structure. GaN grains of the size less than $20-30 \mathrm{~nm}$ always contain stacking faults: the structure of such grains is a combination of hexagonaland cubic-type layers. The density of stacking faults in the smallest GaN grains $(2-4 \mathrm{~nm})$ is very high and the diffraction patterns of such materials resemble those of the cubic-sphalerite phase ( $3 \mathrm{C}$ polytype). The appearance of the cubic-like structure of $\mathrm{GaN}$ is apparently the result of a generation of stacking faults in the original wurtzite lattice. In this work, based on the diffraction data collected in situ during densification of $30 \mathrm{~nm}$ nanocrystalline GaN powder under pressures up to $40 \mathrm{GPa}$, we discuss the mechanism of generation and subsequent relaxation of strains formed in the crystallites. Our analysis is based on a comparison of the experimental diffraction patterns and the intensity profiles calculated theoretically for models of nanocrystalline GaN with different structure disordering and crystallite shapes. 


\section{Experimental procedures and data evaluation}

The nanocrystalline GaN powders were synthesized by pyrolisis of the gallium amide precursor [1]. The densification of the powders with a grain size of $30 \mathrm{~nm}$ and smaller was investigated using the diamond anvil cell (DAC) with pressures up to $40 \mathrm{GPa}$. The experiments were performed without a pressure medium, i.e. under so-called isostatic pressure conditions. The diffraction data were collected in the energy dispersive geometry using gold as the pressure marker. Our typical diffraction patterns are shown in Fig. 1.

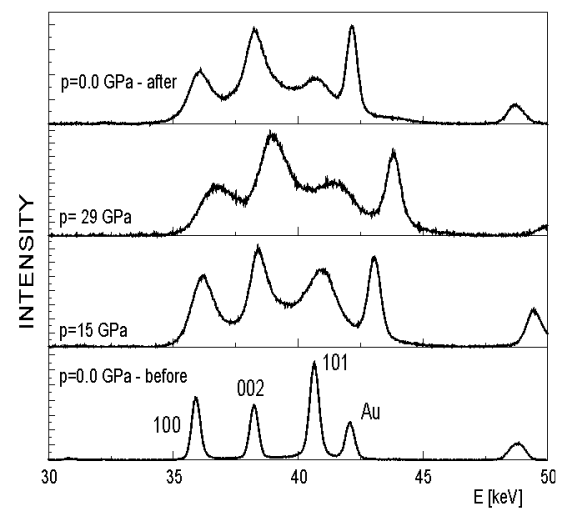

Fig. 1. Experimental diffraction patterns of $30 \mathrm{~nm}$ diameter GaN crystallites obtained under different external pressures.

Elaboration of the diffraction data of GaN nanocrystals was based on tracing the changes in the width of the individual Bragg reflections with a change in the external pressure. The experimental diffraction patterns were interpreted by comparing them with those calculated theoretically for grains with different shapes and sizes based on the Debye functions [2]. We assumed that broadening of the Bragg reflections of a GaN polycrystal exposed to an external pressure may originate from: (i) a presence of microstrains, (ii) changes of grain size and shape, and, (iii) generation of a one-dimensional disorder. The origin of macrostrains generated in $\mathrm{SiC}$ and $\mathrm{GaN}$ nanocrystals was discussed recently in Refs. [3-5].

\section{Results and discussion}

Figure 2 shows a broadening of the Bragg lines determined experimentally for $30 \mathrm{~nm}$ nanocrystalline GaN as a function of pressure for three reflections: 002, 100, and 101. With an increase in pressure, after the initial increase the broadening tends to level off at the pressure about 10 Gpa (Fig. 2). Apparently at the beginning of densification the reflection broadening is due to local strains at the contacts between the crystallites. As the densification process progresses, 


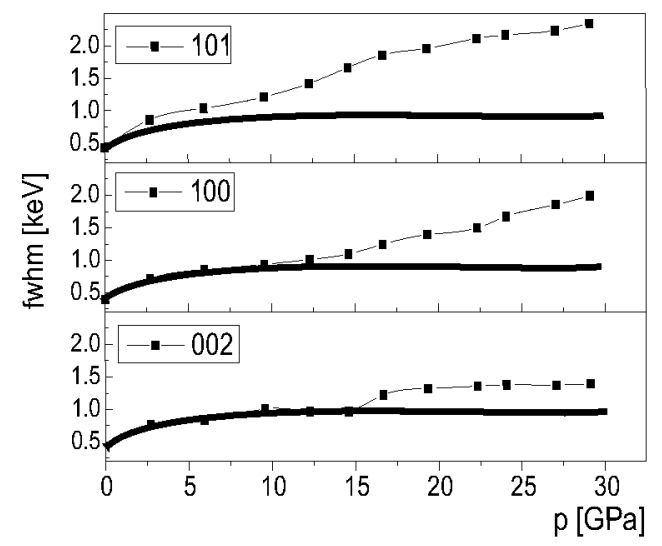

Fig. 2. Change of the broadening of individual Bragg reflections with isostatic pressure for $30 \mathrm{~nm}$ GaN nanocrystals. Squares - experimental results, solid lines - estimated dependence of broadening on pressure due to microstrains.

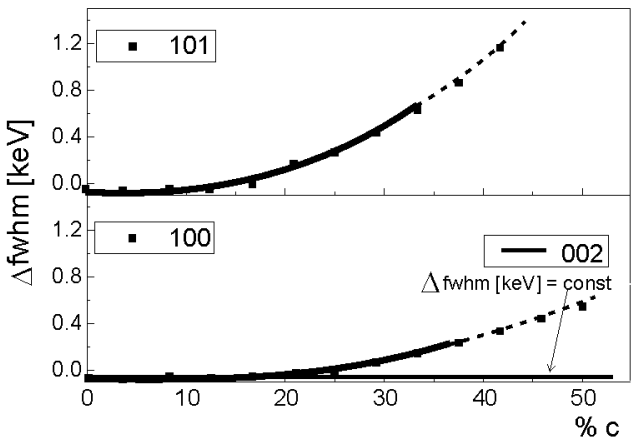

Fig. 3. Theoretically predicted dependence of the broadening of the Bragg reflections on the content of cubic phase in $12 \mathrm{~nm}$ GaN nanocrystals.

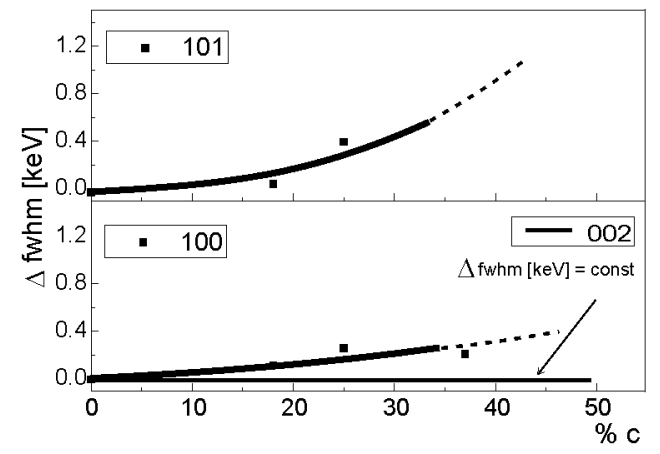

Fig. 4. Theoretically predicted dependence of the broadening of the Bragg reflections on the degree of one-dimensional disordering (randomly distributed single cubic layers in $2 \mathrm{H}$ matrix structure) in $12 \mathrm{~nm}$ GaN nanocrystals. 
the number of local contacts between the crystallites increases, the area of the contacts increases, and in well-densified material a further increase in the applied pressure may be expected not to lead to a further increase in microstrains at the contact points between the crystallites. The broken line in Fig. 2 is an approximate dependence of the broadening due to microstrains (effect (i), c.f. Sec. 2) with an increase in the applied pressure. After the initial leveling off, the total broadening of the Bragg lines significantly increases with an increase in pressure. The difference between the experimentally measured total broadening of the individual reflections and that originating from microstrains (solid and broken lines, respectively, in Fig. 2) can be interpreted as the result of a presence (increase) of one-dimensional disordering and/or changes of the grains shape and dimension.

The effect of the crystallographic defects (stacking faults) and the grain size and shape on the diffraction pattern was determined by numerical simulations $[6,7]$. Broadening of the Bragg lines by a presence of stacking faults in the wurtzite matrix is shown in Figs. 3 and 4. As can be seen, no distinct difference in broadening between grains with different distribution of stacking faults is observed. The effects of the grain size and shape are shown in Figs. 5 and 6. A distinct

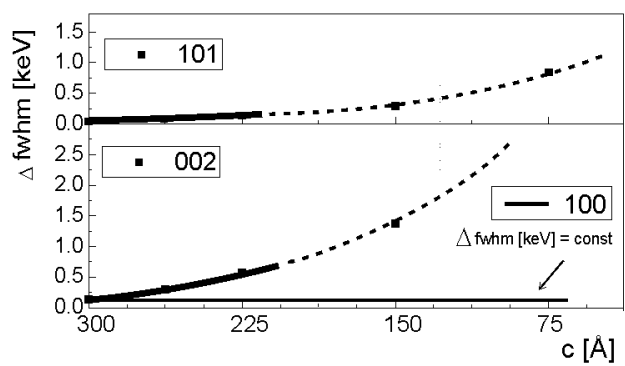

Fig. 5. The calculated effect of the change of platelet-shaped GaN nanocrystallites on broadening of individual Bragg reflections, $a=300 \AA$.

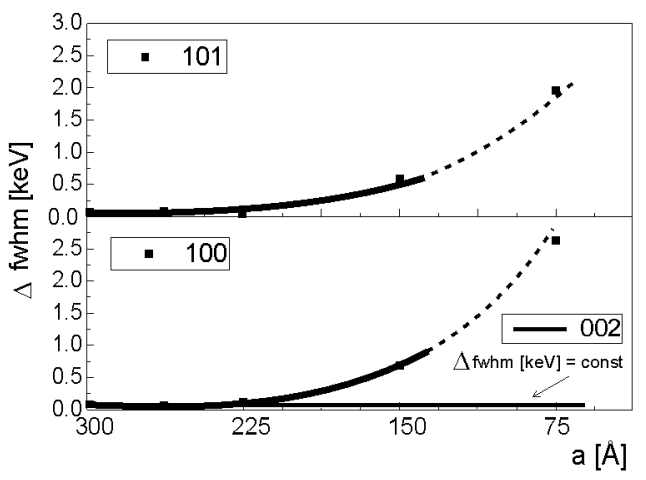

Fig. 6. The calculated effect of the change of needle-shape of GaN nanocrystallites on broadening of individual Bragg reflections, $c=300 \AA$. 
difference between platelet- and needle-shaped grains exists: in the first case no effect of the size on broadening for 100 reflection, in the second for 002 reflection exists. The relative changes in broadening of the three Bragg reflections, 002, 100, and 101 , that result from a presence of stacking faults are very distinctively different from those caused by a change of the shape of the crystallites. That allows to distinguish between the three potential sources of broadening of the Bragg reflection. The comparison of the experimentally determined changes of the reflections broadening (Fig. 2) with those calculated theoretically (Figs. 3-6) leads to the following conclusions concerning the mechanism of densification of GaN nanocrystals under high external stress:

1. For pressures up to $15 \mathrm{GPa}$ the local stresses lead to generation of a one-dimensional disordering in the original wurtzite structure of $\mathrm{GaN}$ : after pressurization the initially hexagonal lattice contains about $35 \%$ of cubic-type layers (compare Figs. 2, 3, and 4).

2. Above 15 GPa no more stacking faults (cubic-layer stackings) are generated. Instead, the GaN grains split into smaller crystallites: the individual grains cleave along the hexagonal layer planes (this is reflected by an increased broadening of the 002 reflection, c.f. Figs. 2 and 5) and along the planes parallel to the hexagonal $c$ axis (compare Figs. 2 and 6).

As can be concluded from the above, relaxation of strain in GaN crystallites occurs through generation of stacking disorder for pressures up to $15 \mathrm{GPa}$, and through splitting the individual crystallites into smaller ones above 15 GPa. Generation of one-dimensional disorder apparently occurs through dislocations that operate along the layer planes. This mechanism proceeds until the density of dislocations (stacking faults) reaches a critical value: the average density of the cubic layers in "fully disordered" crystallites is about $35 \%$. That means that during densification under the pressure of $15 \mathrm{GPa}$ one third of the hexagonal layer converts into cubic ones. At higher pressures the accumulated local strains can be relaxed only through formation of a more compact packing of the individual grains which occurs by breaking the grains into smaller crystallites.

Based on our theoretical calculations of broadening of the Bragg lines for different stacking fault contents and the grain size and shape we conclude that after densification under $30 \mathrm{GPa}$ the starting GaN powder of $30 \mathrm{~nm}$ grain size contains $35 \%$ of cubic layers and the initially spherically shaped grains split into anisotropic, plate-like crystallites. The final shape of the crystallites is $10 \mathrm{~nm}$ in the direction perpendicular to the hexagonal [001] axis and $7 \mathrm{~nm}$ along the axis. Based on this conclusion we built a model of plate-like shaped GaN nanocrystal with $35 \%$ of cubic-type layers and then predicted theoretically the diffraction pattern of such crystallites. A good match of the theoretical and experimental (obtained after densification of $30 \mathrm{~nm} \mathrm{GaN} \mathrm{powder)} \mathrm{results} \mathrm{was} \mathrm{obtained} \mathrm{(Fig.} \mathrm{7).}$

The pressure-induced broadening of the Bragg reflections determined for GaN with grains 2 to $30 \mathrm{~nm}$ in diameter is shown in Fig. 8. As can be seen, 


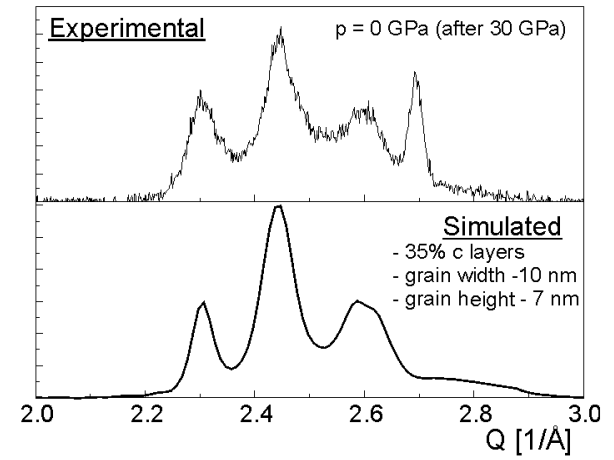

Fig. 7. Diffraction patterns of disordered GaN nanocrystals. Upper curve: experimental pattern of $30 \mathrm{~nm}$ GaN powder obtained at $p=0$ after densification under $30 \mathrm{GPa}$ pressure. Lower curve: theoretical curve obtained for platelet-like grains of $10 \mathrm{~nm}$ in lateral dimension and $7 \mathrm{~nm}$ thick (in [00.1] direction) and containing 35\% of cubic layers.

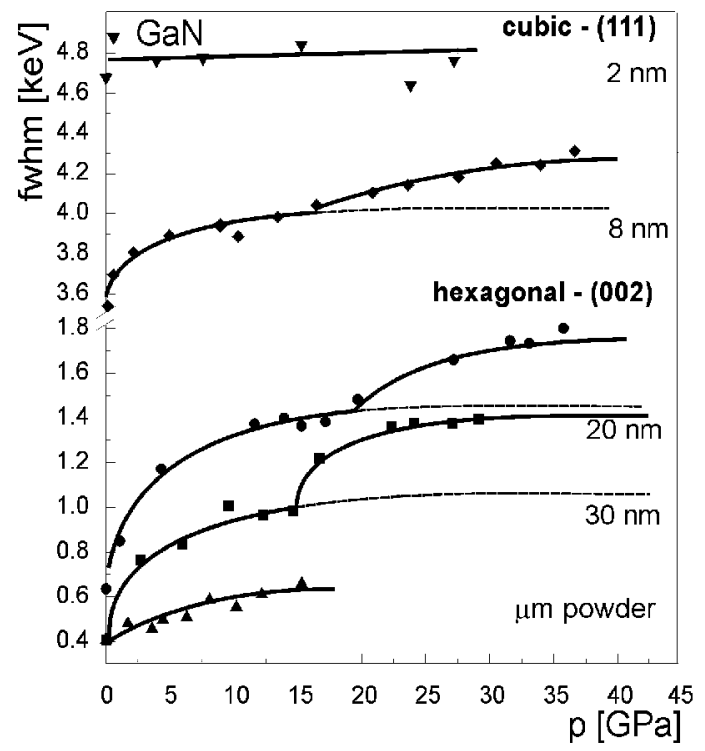

Fig. 8. Dependence of the broadening of the Bragg reflections (111 for cubic, 002 for hexagonal structure) on pressure for different size grains. Symbols and solid lines experimental results, broken lines — estimated (extrapolated) dependence of broadening due to microstrains.

before densification the total broadening of the Bragg reflections is larger for smaller grains. Upon compression under external pressure, the broadening changes (increases) more for larger grains than for the smaller ones. This is consistent with the fact that, in the starting materials, the concentration of stacking faults is 
higher in smaller grains. Based on the comparison of the experimental data with our numerical simulations of the diffraction patterns we conclude that the smallest grains $(2-3 \mathrm{~nm})$ contain about $35 \%$ of the cubic layers (stacking faults). The concentration of the faults decreases with an increase in the grain size and pure hexagonal phase exists in grains of $30 \mathrm{~nm}$ in size or larger. As a consequence, the number of stacking faults that can be formed depends on the size of the grain. For the smallest crystallites the broadening of the Bragg reflections does not change under pressure at all (Fig. 8).

\section{Summary and conclusions}

Under high isostatic pressure generation and relaxation of strains occurs in nanocrystalline GaN powders. At lower pressures (up to $15 \mathrm{GPa}$ ) the strains are relaxed through generation of stacking faults (cubic-type layers in the hexagonal matrix). The maximum concentration of the faults that can be formed in the grains is about $35 \%$. At higher pressures no more stacking faults can be generated and the strain relaxation occurs through splitting the crystallites along the hexagonal layer planes into smaller particles, which increases the total area of the contact between the grains and reduces the internal stress in the structure.

\section{Acknowledgments}

This work was supported by the State Committee for Scientific Research, grant PBZ/KBN-013/T08/30, the Polish-German project POL-00/009 and HASYLAB project I-99-068. A part of the effort was supported also by the EC grant "Support for Centers of Excellence" No. ICA1-CT-2000-70005. The support from the Office of Physical and Biological Research of NASA is gratefully acknowledged.

\section{References}

[1] J.F. Janik, J.R. Wells, Chem. Mater. 8, 2708 (1996).

[2] B. Bondars, S. Gierlotka, B. Palosz, S. Smekhnov, Mater. Sci. Forum 166-169, 737 (1993).

[3] B. Palosz, S. Gierlotka, E. Grzanka, K. Akimow, R. Pielaszek, P. Biczyk, A. Grzegorczyk, S. Stel'makh, U. Bismayer, J.F. Janik, Mater. Sci. Forum 378-381, 735 (2001).

[4] E. Grzanka, B. Palosz, S. Gierlotka, R. Pielaszek, K. Akimow, U. Bismayer, J.F. Janik, Annual Report Hasylab 2000, p. 481.

[5] E. Grzanka, B. Palosz, S. Gierlotka, R. Pielaszek, K. Akimow, U. Bismayer, J.F. Janik, Annual Report Hasylab 2000, p. 483.

[6] R. Pielaszek, S. Gierlotka, S. Stel'makh, E. Grzanka, B. Palosz, Defect and Diffusion Forum 208-209, 189 (2002).

[7] J-W. Hwang, J.P. Campbell, J. Kozubowski, S.A. Hanson, J.E. Evans, W.L. Gladfelter, Chem. Mater. 7, 517 (1995). 